\title{
La ricezione di Pavese nei narratori spagnoli del secondo Novecento
}

\author{
Piero DAL Bon \\ Universitat de Barcelona \\ dalbon@ub.edu
}

\begin{abstract}
Riassunto
Cesare Pavese ha incontrato in Spagna lungo la seconda metà del secolo scorso, una prolungata accoglienza, ora generalizzata e intensa ora più puntiforme e occasionale, sempre comunque molto vivida. Il contesto nel quale per la prima volta irrompe il nome di Pavese è quello della Spagna degli anni Cinquanta. Nel saggio si studia l'influenza dell'autore italiano su narratori e lirici di diverse generazioni; tra di essi i fratelli Goytisolo, Gabriel Ferrater e altri minori.
\end{abstract}

Parole chiave: Ricezione, Pavese, scrittori spagnoli, anni Cinquanta.

The reception of Pavese in the Spanish narrators of the second Novecento

\begin{abstract}
Cesare Pavese found in Spain a wide reception during the second half of the twentieth century, both in a general and intense and particular and specialized ways. The first time that appears the name of Pavese in Spain is during the fifties. This essay studies the influence of the Italian writer in narrators and poets of different generations; such as the Goytisolo brothers, Gabriel Ferrater and other minor writers.
\end{abstract}

Key words: Reception, Pavese, Spanish writers, the fifties.

Dal Bon, Piero. 2011. La ricezione di Pavese nei narratori spagnoli del secondo Novecento. Cuadernos de Filología Italiana, nº extraordinario: págs. 145-157.

Insieme antico e moderno, rurale e cittadino, Pavese rappresentò per gli intellettuali spagnoli il modello di un narratore combinabile sia con gli archetipi della tradizione nativa, sia con i modelli più all'avanguardia del romanzo esistenzialista e del nouveau roman che presentavano e rappresentavano una soggettività esposta a 
tutti i traumi e i disastri di una contemporaneità alienata. Il contesto nel quale per la prima volta entra il nome di Pavese (concordi nel segnalarlo tra i modelli decisivi per gli autori che esordiscono negli anni cinquanta sono numerosi storici del periodo, tra cui Sanz Villanueva (1980:132-183), Sobejano (1970: 229), Corrales Egea (1971: 197), Jordan (1990: 125) è quello di una Spagna degli anni cinquanta in profondo rinnovamento sociale, culturale, nella direzione di una rottura dell'isolamento intellettuale che la separava dal resto dell'Europa. Irruppe sulla scena letteraria del paese una nuova ondata generazionale le cui parole d'ordine furono realismo, oggettività ed impegno (il vocabolario autocritico di Matute e Martín Gaite, Juan e Luis Goytisolo, Aldecoa e Fernández Santos abbonda di questi termini e dei loro più immediati sinonimi).

Un'imprescindibile funzione di rottura storica, un ruolo decisivo nell'affermazione dell'egemonia, quasi incontrastata di un estetica neorealista la ebbe la penetrazione del nuovo cinema italiano (lo documenta il saggio di Fernández Fernández 1992 che illustra con dovizia di dati le tappe della massiccia influenza neorealista): il prestigio di De Sica, Rossellini, Zavattini aprì la breccia attraverso la quale si infilarono successivamente Pratolini, Vittorini, Levi, e altri, favorendo un insolito interesse per quanto si era andati facendo in Italia. Nel caso di Cesare Pavese giovò certo anche la suggestione della sua fitta attività di americanista, come afferma Bravo (1985: 125): «El interés que por Faulkner demostró Pavese fue un factor importante para su estimación». Spesso il nome dei due autori compare associato in dichiarazioni in cui si rievocano gli anni di apprendistato e le letture dei romanzieri di questa generazione: la creazione di un universo narrativo occluso e tragico, il motivo dell'inesorabilità del destino, ebbero un notevole impatto sulla vocazione al registro cupo di alcuni scrittori del tempo. La narrativa americana aveva due illustri difensori nelle persone di Castellet e Juan Goytisolo: il critico catalano ebbe il merito di diffondere e difendere i principi della behaviour novel (il cosidetto romanzo senza autore, la cui tecnica fondamentalmente basata sull'understatement, su di un'estetica dell'implicito, sulla laconica allusività, avrebbe avuto nello stesso tempo il pregio di aggirare le insidie censorie e di richiedere una collaborazione concettuale più intensa da parte del lettore; Castellet 1957), il romanziere quello di ribadirli (Goytisolo 1959), allargando le sue osservazioni teoriche alla letteratura italiana: Vittorini e Pavese vennero considerati gli eredi più prossimi dei romanzieri americani.

La lezione di Pavese agisce sullla prima produzione narrativa di Luis Goytisolo: a metterci sulle tracce di una possibile comunanza di risultati tecnico-stilistici e sull'analogia di alcuni nuclei tematici forti nelle traiettorie dei due autori aiutano sia dichiarazioni autocritiche dell'autore sia commenti altrui. Las afueras (L. Goystisolo, 1958), titolo dalla semantica polivalente (può indicare, come suggerisce Fernando Valls nella densa introduzione, un luogo geografico preciso, ed essere insieme, metafora esistenziale di vite marginali, periferiche), mostra nell'inclinazione verso un realismo critico e nell'interesse verso la società agricola un'evidente influenza pavesiana, suffragata da numerose dichiarazione dell'autore. Nella prima testimonianza (Valls 1958: 4) il giornalista riporta le dichiarazioni attraverso l'indiretto libero riferito, da esse veniamo a conoscenza del fatto che, al momento della stesu- 
ra del suo primo romanzo, i modelli del narratore catalano erano Faulkner, Hemingway, Dos Passos, Pavese, Pratolini, Vittorini: l'autore torinese pare godere di una stima tutta particolare se è vero che lo si giudica il miglior romanziere italiano della sua generazione. Maggiori dettagli si evincono dall'intervista più ravvicinata nel tempo (contenuta nella tesi di dottorato di Eugenio Asensio Solaz, La presencia de Cesare Pavese en los narradores del medio siglo, discussa nella UB, nel 1991, e inedita). Ribadito l'interesse di quegli anni per Pavese, Goytisolo questa volta ne spiega le ragioni profonde, obbedienti a due fattori, «era un'interesse logico, perchè descriveva una realtà molto più affine alla nostra, ti parlava quasi della stessa cosa. Quello che mi fece maggiormente impressione fu la forma di trattare la realtà di un paese tanto simile alla Spagna di allora» (Goytisolo 1959: p. 89). Le somiglianze possiamo intuirle ed indicarle sinteticamente, nella presenza di una dittatura e nella frequente tematica rurale, messe in antitetico contatto con quella cittadina. Nell'affermare che il suo contatto diretto con Pavese avvenne attraverso le edizioni argentine, celebri per le loro malefatte, l'autore barcellonese assegna le proprie preferenze all'ultimo romanzo testamento del piemontese, considerato come un'elaborazione più sofisticata del trattamento dei tempi narrativi (il racconto che assomiglia ad un altro racconto, le analepsi etereodiegetiche che slargano il campo del narrato, rammemorando il cammino di iniziazione del protagonista-narratore) e delle relazioni tra i personaggi. Tra le altre osservazioni spicca la nota formale in cui l'apparente semplicità stilistica della prosa pavesiana viene modellata dalla sua capacità di fare tabula rasa di fastidiose incrostazioni letterarie, dalla sua prensile aderenza alla realtà. Ma andiamo al testo, al primo dei sette capitoli che compongono Las afueras, citando dall'edizione a cui si rimanda nella biblografia finale.

L'inizio presenta una situazione narrativa, sceneggiata direttamente, di un ritorno in campagna: il protagonista, Víctor, non viene introdotto da didascalie chiarificatrici da parte della voce esterna, ma presentato nel farsi di un'azione, nella interna mobilità prospettica di un cammino in salita, dalle cui successive agnizioni visive e auditive, anticipatrici, il lettore inferisce subito la decettività dell'esperienza a cui sta approssimandosi: «Desde allí, desde el rastrojo la casa ... ofrecía el aspecto de siempre. Sólo que ahora la chimenea no humeaba ni se oían voces ni ladridos y no había ropa blanca secándose al viento en los alambres tendidos sobre el pretil de la era» (Goytisolo 1958: 56). Lo spazio mitificato dell'infanzia, il luogo del ricordo sublimante, rivela da subito la propria natura degradata, devitalizzata. La suggestione atmosferica viene restituita da uno stile evenemenziale, registrante, oggettivo, che non lascia spazio alcuno ai sondaggi analitici nell'interiorità del personaggio, pedinato dal narratore-spettatore e contemplato nell'evidenza esteriore dei movimenti e dei gesti. Durante l'intero corso della narrazione nulla ci viene spiegato dei vari attori, nessun dato caratteriale risulta esplicitamente dichiarato, ma rivediamo tutto dal progressivo sdipanarsi dell'azione, come nell'allusiva oggettività, senza residui didascalici, del finale:

Al poco, la niña asomó la cabeza y durante algunos segundos miró calladamente su espalda encorvada sobre el hogar. Luego avanzó de puntillas hasta dejar el cántaro en el extremo de la mesa más cercano a la entrada. Pero Víctor no se volvió 
entonces ni cuando se alejaron aquellos pasitos breves y sigilosos. Miraba el fuego, los grandes leños que ardían y chisporroteaban rezumando savia burbujeante. Eran ramas de algarrobo (Goytisolo 1958: 111).

Come recita la prefazione (che parafraso), dietro la superficie della storia se ne intuisce un'altra, sotterranea, quella dell'inquietudine spaesata di Víctor che non riesce a trovare il proprio luogo nel mondo, quella di un'arrovellata frustrazione senza scampa o sfogo che non si pacifica a contatto degli scenari che furono i suoi, quelli della sua infanzia, evocati da un sobrio lirismo (analogo a quello di Pavese):

Del barranco subía un húmedo aroma de resinas, de hierba caliente. No se escuchaba otro ruido que el seco chasquear de las cortezas, el escurrirse de los lagartos entre la hojarasca. El bosque se extendía por las colinas, suavemente revuelto, quieto, como apagado bajo aquel cielo blanquecino que ablandaba las bojas (Goytisolo 1958: 66).

Capita così che l'azione descriva i tentativi di recupero di una condizione infantile, come nelle ruberie di frutta nei vigneti, e capita che il dialogato, sciolto, senza more, veda affiorare il rimpianto per una condizione che la vita adulta, nel caso del protagonista Víctor, legata ad un presente cittadino, ha perduto. L'incontro con gli amici di allora attira il rimpianto per uno stato che era poi, secondo una dialettica pavesiana, già proteso verso il proprio superamento: il viaggio nello spazio diviene un viaggio nel tempo, a ritroso, nei luoghi mitici del proprio passato agreste: «[...]Y sus rincones particulares, aquellos que ni los mejores amigos conocían: el fondo de un torrente, lugar sagrado, escondido, aquel lecho de arena blanca [....]» (Goytisolo 1958: 104).

La vocazione fallimentare del protagonista, l'asfissia del suo destino, viene tracciata con mano sicura, nella ricerca di uno sbocco salvifico che rimane di là dal divenire, fino ad un finale di rinuncia. È in questi paraggi tematici che il minore dei Goytisolo può aver sentito la suggestione della narrativa di Pavese, il cui complesso gioco di antinomie con il suo sicuro retroterra filosofico viene semplificato in una più elementare e irrigidita antitesi. Se l'infanzia nel primo capitolo è il tempo assente ed invocato, nel sesto diventa fuoco prospettico, centro narrativo: l'iniziazione del «niño» Bernardo alla realtà adulta, la scoperta della verità delle cose passa attraverso la rivelazione dell'orrore della violenza. L'attacco del racconto è mimetico, diretto; cala il lettore nel vivo di una scena, senza mediazioni diegetiche (ricordiamo Paesi tuoi) «¿Qué les has dicho? -Nada-¿Nada?» (Goytisolo 1958: 218). Poi il racconto, formalmente, sta tutto nel procedere scorciato e nei passaggi da una sequenza all'altra «¿Adónde vas, Bernardo?/ Patrach estaba en el bar de abajo» (Goytisolo 1958: 222), e nella radente elitticità delle microsequenze sintattiche, «Luego viento en la calle»: la lingua di Las afueras è fatta tutta di una sprezzata colloquialità, di un dettato sincopato e rasoterra, e di un montaggio di spezzoni narrativi sceneggiati direttamente, con incisiva aderenza all'essere in situazione dei vari attori.

Nel romanzo sucessivo, Las mismas palabras (Goytisolo 1961), l'autore sposta la propria attenzione narrativa sulla giovane borghesia catalana. Alta è l'ambizione 
architettonica, se è vero che tre storie parallele e indipendenti vengono raccontate, con montaggio simultaneo, in sette capitoli di varia lunghezza. I modelli narrativi sembrano essere, questa volta, il Cela de La Colmena e il Sánchez Ferlosio de El Jarama, dico per l'ampia polifonia corale, per la stipata presenza di un dialogare svariante che spesso diventa chiacchiera brillante ma elusiva. La frequente presenza della finestra (motivo segnalato da Basile 1985) allude ad un motivo simbolico, di possibile ascendenza pavesiana, quello della fuga o dell'uscita, impossibili ma desiderate, che soggiace a tutto l'anfrattuoso prolungarsi del narrato, il fatto è che «todos se aburren, todos divagan, ninguno desemboca». La cieca quotidianeità di vite senza senso viene testimoniata con corrosiva impassibilità da un Goytisolo che ha assunto oramai a propri temi cruciali l'apatia depressiva, lo snervante tedio che frena qualsiasi slancio di intraprendenza volta a costrurisi un'etica o un destino. Si tratta di un un'occlusa iteratività vitale: un rigiro d'esistenze, che si perdono in vuoti intrecci mondani, tra festine e gite sulla costa, psicofarmaci e alcool, sono documentate da un romanzo le cui parole d'avvio ne marcano implacabilmente il corso: «Las mismas palabras, los mismos gestos, todo volvió a suceder igual que antes, cuando Rafael salía con Berta y los amigos y no se acostaban hasta la madrugada [....]» (Goytisolo 1961: 3).

Se nell'ultima intervista sopra citatata Luis Goytisolo indicava nelle somiglianze tra Italia e Spagna gli spunti che stimolarono il suo interesse verso Pavese, questo può essere vero anche per la analogia di scenari in cui si muoveva una borghesia medio-alta edonista, svagata e disicantata. Possibili ipotesti della narrazione sono i romanzi di Pavese che rappresentano criticamente la società borghese: Il diavolo sulle colline e soprattutto Tra donne sole. Particolarmente la configurazione del personaggio di Rafael, prossimo al Víctor de Las afueras, è a riportare alla mente suggestioni pavesiane, individuato in una smaniosa, irrequieta solitudine urbana; sono i suoi gesti, i suoi movimenti a parlare di lui, in una densa oggettiva allusività:

Sacudió el cigarillo. La ceniza cayó al lago con un seco chirrido y se hundió en la verdosa claridad del agua. La siguió con la vista según se disgregaba y reducía en su turbio descenso hacia las pálidas piedras del fondo [...]» (Goytisolo 1961: 16).

Con paradigmatico movimento regressivo alla sua solitudine urbana si contrappone l'antitesi rurale, indelebilmente legata all'infanzia. Il capitolo 20 segna il momentaneo arresto, acronico, della temporalità perversa dell'età matura, per accogliere la revivescenza epifanica di un passato che si fa presente, che irrompe nel presente, cancellando la consequenzialità cronologica:

Parado en la pérgola, contemplaba el valle oscurecido, los campos de su infancia y su juventud, las colinas. Se acodó en la baranda, escrutó el paisaje, los contornos imprecisos en aquella hora de calma, y era como volver a vivir lo ya vivido, a escuchar palabras ya pronunciadas, voces de otros tiempos [...] (Goytisolo 1961: 139).

L'amara autocoscienza di un vuoto vitale, di una latitanza della pienezza, unisce Rafael alla decoratrice Julia, anch'essa caratterizzata da un'insoddisfatta noia che la 
porta a concludere: «Es inútil engañarse. Pasa un día y otro y una semana y otra y un año y todos los días son iguales y nunca cambia nada. Hoy un cóctel, mañana una cita, el domingo la playa [...]» (Goytisolo 1961:48); parole che potrebbero uscire da una dialogo di Tra donne sole, prototipo di romanzo mondano, ricco in situazioni sociali e dialoghi. Questi, introdotti da scarne didascalie, sono gestiti dal narratore esterno con mano destra e fedeltà sociolinguistica, nel riprodurre la lingua della conversazione del gruppo sociale rappresentato. Il passo del racconto procede attraverso sottili schermaglie dialogiche, come questa, senza che niente succeda, se non un astioso attorcersi di ciascuno su sè stesso: i movimenti sono illusori, lo spazio tende a configurarsi come prigione compreso quello cittadino barcellonese, circondato da colline. Plausibili, e comprovate da abbondanti riferimenti critici e autocritici paiano anche le tangenze tra Pavese e Juan Goytsolo, forse l'intellettuale con la vocazione più proiettata verso l'estero, piu curiosa e disponibile verso le varie elaborazioni letterarie che si venivano creando fuori dalla Spagna, al limite del camaleontismo. A sottolineare questa propensione alla vorace assimilazione di esperienze estetiche contemporanee fu Pere Gimferrer nell'introduzione ai due volumi delle opere complete di Goytisolo (1978) (da cui provengono le citazioni contenute nel presente saggio). E fra i nomi che fanno capolino nelle autorevoli parole del critico compare anche quello di Pavese, modello stilistico e più generalmente intelllettuale:

A ello se añade el influjo de los escritores behavioristas, principalmente de Hemingway, Faulkner (en la medida en que sea lícito considerarle como tal), y de modo particular Pavese, que, para un intelectual de nuestra península, representaba, en cierto modo, un modelo ideal: heredero, por su técnica, de los americanos, se hallaba vinculado a una sociedad no muy desemejante de la que se había vivido en España; simpatizante con el marxismo, separado luego de él como encarrnación del dilema entre las convicciones políticas socialistas y la problemática personal, entre programa e individuo, entre filiación burguesa y actividad revolucionaria e incluso, genéricamente, entre intelectual puro y hombre de acción (Gimferrer 1978: 89).

Gimferrer limita l'azione di influenza dell'autore torinese (ma l'allarga a comprendervi anche Las mismas palabras e Tormenta de Verano) al periodo che precede immediatamente la svolta sperimentale e deflagrante di Señas de identidad, opere che si collocano nell'alveo di un realismo, di forti intenzioni critico-demistificatrici, simile negli obbiettivi polemici a quello de Las mismas palabras: parlo di La isla (1961) e Fin de Fiestas (1962). Ad un arco cronologico più ampio fa, invece, riferimento Goytisolo stesso, da quanto possiamo dedurre, dal citato saggio contenuto in Problemas de la novela e dalla data di un intervista rilasciata per la rivista lndice (Goytisolo 1958: 118) in cui dichiara:

En España las cosas son mucho más claras y el novelista sabe muy bien lo que debe escribir. La dificultad consiste, naturalmente, en encontrar la manera. Para ello creo que debemos aprovechar la lección de los grandes novelistas italianos como Vittorini y Pavese (Goytisolo 1958: 118). 
e dalle affermazioni autocritiche retrospettive, che, prendano in considerazione gli anni che vanno dal 1955 al 1960 :

A mí me influyeron bastante en esta etapa los italianos que habían crecido en la época del fascismo. Me interesaban mucho por la similitud de problemas con respeto a lo que nosotros sentíamos sobre el régimen español. Leí muchísimo, y los que más me influyeron fueron Pavese, Vittorini y el libro de Carlo Levi «Cristo se detuvo en Eboli» (Lázaro 1982: 154).

Più o meno gli stessi nomi ritornano in pagine autobiografiche anche cronologicamente più prossime: ridisegnando la traiettoria della propria parabola narrativa, Juan Goytisolo dice:

Tras la fallida tentativa de novela social con "la resaca", tantearé modalidades del reportaje narrativo y relato breve que siguiendo las huellas de Rocco Scotellaro, Vittorini y Pavese, desenvolveré con mayor o menor ventura de Campos de Nijar a Pueblo en marcha (Goytisolo 1992: 22).

È interessante constatare come la suggestione di Pavese, congiunta con quella più penetrante di Vittorini, può aver contribuito ad autorizzare l'apertura ad un tipo di testo, la cui vocazione principale è quella della scoperta dell'antropologicamente altro, del selvaggio e dell'esotico, nelle modalità archetipiche di un viaggio nei territori semidesertici dell'Andalusia. A questo periodo, fervido di sperimentazioni in tutti i campi della scrittura, risale la raccolta di racconti Para vivir aquí (1960) a cui allude sopra l'autore, racconti rigorosamente oggettivi condotti da un narratore interno, che varia di narrazione (otto in totale), muovendosi sempre in scenari di forte verosimiglianza (e magari in Aquí abajo nella sua storia di una redenzione politica si può sentire la lontano eco de Il compagno, romanzo che nella sua esemplare traiettoria verso un definitivo impegno dovette certo colpire gli intellettuali del tempo). Il taglio dei racconti è sempre rapido, dall'avvio senza esitazioni, il suo passo regolato da una sintassi scarna, svelta, tutta azione, in cui Goytisolo non lascia posto a compiacimenti estetizzanti, e mira ad un'adesione stringente al mondo rappresentato. Il primo romanzo con tracce del piemontese, La isla potrebbe rivelare affinità con Tra donne sole. Come la Clelia Gitana della narrazione pavesiana la Claudia di Juan Goytisolo ritorna nel luogo d'origine e lo fa in mutate condizioni sociali: umili proletarie, hanno raggiunto una condizione economica che le permette di frequentare il mondo della alta classe, da cui ricavano il senso di una società amorale e vacua, di un teatrale girotondo mistificato in cui domina il disvalore. Comune ai due testi è l'impostazione tecnico-formale, nella presenza di un narratore interno, omodiegetico, le protagoniste stesse, da cui viene anche il discreto piglio antiborghese, che emerge non dalla presenza di commenti espliciti ma dalla dispersiva sequela di incontri e dialoghi. Ma le somiglianze si fermano qui.

Una maggiore quota di osservazioni e parallelismi la propone l'opera successiva Fin de Fiesta. Simile a Las afueras per lo statuto ibrido del genere, tra romanzo e racconto, ques'opera presenta quattro narrazioni, «variazioni su di un unico tema», che è sempre una crisi matrimoniale, provocata dall'intromissione di una terza per- 
sona, crisi matrimoniale che alla fine rientra, in un apparente ritorno allo normalità. Se la presenza dei consueti motivi del tedio, dell'apatia irresoluta e del vuoto borghese rimandano ad una ipotetica koinè esistenzialista e fitzgeraldiana che raggrupperebbe gran parte dei romanzieri europei di questi anni, particolare interesse ai fini di quanto andiamo trattando lo mostrano i capitoli I e IV. Quest'ultimo presenta un singolare triangolo, in cui alla coppia si aggiunge un amico d'infanzia del marito, invitato a trascorrere un soggiorno estivo nella casa di campagna degli sposi: il che ci rimanda, ovviamente, a La Spiaggia. Un'identica strategia narratologica lega le due narrazioni, la prima persona dell'invitato che rammemora, sobriamente, laconicamente, il periodo estivo. In entrambi i testi la trama è assente, e le narrazioni scorrono via in un'apparente vacuità d'eventi. Scapoli e maturi, connotati da tratti di intellettuale disincanto, amanti della solitudine, i due narratori-protagonisti, all'arrivo, si trovano nel bel mezzo di una bonaccia matrimoniale, di cui, con curiosità un poco morbosa, cercano indizi e cause, pur nella reticenza dei coniugi. Comune è il ruolo della donna che sovrappone come un diaframma nella relazione tra gli amici d'infanzia: tra l'invitato e la sposa si crea un rapporto di confidenza privilegiato, un solo alluso innamoramento, che si specchia, in un sottile gioco di sdoppiamento, nella giovane figura di due personaggi secondari singolarmente simili, Berti e Jorge, latori di una passione amorosa impossibile che li introduce al dolore. Nell'amaro eludersi e inseguirsi senza mai raggiungersi della coppia di amici, i due narratori occultano l'impossibile ritorno ad una condizione precedente, ne La spiaggia brevemente recuperata nel secondo, il più ilare, degli undici capitoli, in Fin de fiestas apertamente, pateticamente rimpianta. Chiaro dev'essere, però, che dove Pavese risolve la crisi nella salvezza rappresentata dalla rinuncia, stoica sublimazione del ricordo, Goytisolo la protrae pessimisticamente:

Su rostro había recobrado la expresión huérfana de la niñez y, con una acuidad que me sorprendió a mí mismo, medí los límites de nuestro desamparo, de Miguel y mío y de Mara- frente a las ofensas de la vida. Bueno - dijo-. Habrá que buscar otro pretexto. Miguel hablaba muy despacio y alumbró calmosamente un cigarillo. Hoy por hoy la fiesta ha terminado (Goytisolo 1992: 181-2).

Amarezza e disincanto contraddistinguono anche la prima delle quattro composizioni di Fin de fiesta: se in quella precedente il centro della rappresentazione era l'età adulta, qui il fuoco narrativo è l'adolescenza, che pur non essendo esplicitamente all'interno del quadro del racconto, tiene nella persona di un ragazzo le redini dela narrazione eterodiegetica. L'espediente prospettico garantisce la messa in rilievo della morosità di un'iniziazione alla vita e ai misteri degli adulti attraverso la scoperta degli intrighi del sesso: l'amicizia tra il narratore e il più adulto Ramón si vede interrotta dall'amore di questo per una donna svedese sposata, il che porta il marito di questa a tentare il suicidio. L'allontanamento dell' amico e lo scoppiare di un'improvvisa violenza, l'entrata della donna nel giro di una relazione morbosamente esclusiva, rompono l'ingenua fantasia trasfigurante del narratore che a questo punto non sarà più lo stesso. Il passaggio da uno stato all'altro, da un'età all'altra viene allusivamente adombrato nella constatazione che segue: «Yo miraba los cipre- 
ses del cementerio, el hotel, las colinas, y comprendí de golpe que mis vacaciones habían terminado» (Goytisolo 1992: 33), in cui il significato di vacanza si allarga, accogliendo anche quello di innocenza, di innocente adolescenza.

Non si può, a questo punto, non pensare ad alcuni racconti di Feria d'agosto, in particolare a La giacchetta di cuoio, a narratore interno, con quell'amicizia tra Pino, il ragazzo, e Ceresa, l'adulto, e la profonda associazione tra sesso e violenza, e non si può non ricordare la profanazione dell'Eden infantile di Primo amore, sempre un racconto a narratore interno scarsamente consapevole, e sempre un racconto iniziatico, di rivelazione del selvaggio attraverso il sesso adulto. La coppia ragazzo-adulto sembra essere un tema pavesiano che ha interessato molto Juan Goytisolo, per quella mescolanza, particolarmente affine al suo dualistico temperamento creativo, di inclinazione al sogno, amplificazione fantastica e attitudine etico-realista, segnalata da vari critici: la struttura dei romanzi della trilogia de El mañana efimero, formata da El circo (1957), La resaca (1958), Fiestas (1958), si costruisce proprio sull'asse di questa coppia che modella la storia di una formazione diventata storia di una deformazione, di una mutilazione e di una ferita. Ne La resaca la vicenda del bambino Antonio è la storia di una disillusione, di un' iniziazione mediata da un tradimento da parte di un adulto, Metralla; in Fiestas -quella di Pipo a dare il diagramma di una parabola che si dirige verso la constatazione della sordida crudezza della realtà, verso l'allontanamento da un'infanzia percepita come un'età mitica, che diventa, però, condanna al non riconoscimento della verità delle cose, cecità ed esclusione, se protratta. È in questo senso che va letta l' affermazione di Fiestas «hay algo más triste que envejecer: es continuar siendo niño», ripresa dal Mestiere di vivere. $\mathrm{E}$ anche in questa intuizione del cruento presente nei rapporti umani, dell'archetipica loro violenza che Juan Goytisolo può essere sentito vicino a Cesare Pavese (Gimferrer 1978: 44-93).

Adolescenza ed infanzia, nella loro potente capacità di stupore e rivelazione critica della realtà storico-sociale, concepite come frangenti temporali decisivi dell'esperienza umana, sono l'oggetto anche della dolorosa contemplazione narrativa di Jesús Fernández Santos, forse il piu pavesiano dei narratori spagnoli, nella raccolta di racconti intitolata Cabeza rapada (1959; si citerà da Fernández Santos 1983): una silloge costituita da quattordici racconti, di varia materia, ora bellica (El sargento, Muy lejos de Madrid, El primo Rafael, Pecados, El final de la guerra), ora rurale (Llegar a más, Hombres, La historia de Juana) ora cittadina (La vocación, El doble), ora, appunto, miranti a raccontare esperienze liminari, proprie dell'infanzia e dell'adolescenza (l'eponimo Cabeza rapada, Una fiesta, Día de caza, Los veraneantes), che spesso s'intrecciano con i precedenti, sempre, pero, all'interno di un codice di verosimiglianza realistica e, nello stesso tempo, di portata allusiva e metaforica nella rappresentazione dell'isolamento e del fallimento delle diverse molecole di una società prostrata e periferica. Una gamma cromatica che tende al grigiore, un'inclinazione alla costruzione di narrazioni che abbracciano situazioni umane emblematiche nel giro di poche pagine, nella brevità che consuma ogni residuo superfluo: Fernández Santos ha costruito una raccolta di racconti di grande concentrazione espressiva, il cui primo movimento creativo sembra essere quello della decantazione e dell'eliminazione di tutto quanto vi può essere di ornamentale. È anche in ques- 
ta estrema economia di mezzi espressivi, nella laconicità di un dettato che non-indugia, che si può riconoscere l'impronta di Cesare Pavese. Le analogie non si fermano qui, ma vanno più a fondo, se è vero che in uno dei primi contributi critici apparsi in Spagna sulla raccolta dell'autore di Madrid, Antonio Vilanova, estrapolava nei suoi rilievi tecnici le seguenti caratteristiche:

[... ] es el suyo un arte preciso y conciso que representa escenas y trozos de vida extraídos de la realidad cotidiana y vulgar y que retrata sentimientos y estados de alma, más por la insinuación y sugestión de los hechos y situaciones que plantea que por la descripción o el análisis de los personajes y de sus problemas (Vilanova 1980: 3409$)$.

una somma di tratti stilistici che appartengono anche all'autore torinese, in un'oggettività che dice non dicendo. E fu proprio Vilanova, tra i primi, a fare il nome di Pavese stesso, attribuendogli non solo il magistero nell'ideazione della raccolta ma anche il ruolo di modello più ravvicinato del racconto conclusivo della silloge. L'avvio del racconto introduce subito il tema, quella di un congedo, di una fine, del declino di una pienezza: «El otoño se anticipó aquel año», le cui armoniche risonueranno sottilmente lungo l'intero corso del narrato, e nell'allusività delle situazioni, evocate con il solo accenno di alcuni tocchi, e nella emblematicità di dialoghi rasoterra:

Entró de nuevo toda orquesta, concluyendo con un toque agudo y prolongado. Bueno, esto se acabó. Parece que nos estaban esperando- se lamentaba la muchacha, pero la gente aplaudía y vino la propina. ¿Bailamos esta última? La despedida [...] Me estoy quedando fría. Entraron en baile, pero el baile pareció rechazarles (Vilanova 1980: 161).

Quello che si consuma, nell'apparente piatta enumerazione di piccoli fatti e parole feriali, è la lenta ma definitiva presa di coscienza di Pablo, il protagonista e portatore di prospettiva del racconto, il quale scopre che un'epoca si è definitivamente conclusa, che qualcosa di sconosciuto si apre, qualcosa di sconosciuto e di amaro. Da qui proviene il fatto che particolari descrittivi che entrano nel campo percettivo del personaggio (svelantesi, come tutti gli altri personaggi, poco a poco, non illustrata da didascalie esterne) emettano vibrazioni particolari:

Bajo la mancha azul del cielo, era la verbena una ampolla de luz bajo otra luz, bajo el haz intermitente del faro. Pablo, en la playa, limpió de arena las piernas de su pareja.Te quiero mucho- repitió la muchacha. Pablo, mirando el halo circular en el que el agua se deshacía con su estrépito, no contestó. Nos vamos. Hace frío. Un poco de humedad. La marea estallaba como una carga, rompiendo paulatinamente todo su frente (Vilanova 1980: 159-160).

Carico di una strategia semantica tutta particolare, è il finale, in cui Fernández Santos gioca le sue carte narrative, puntando tutto sull'explicit senza commento, sul simbolismo sospeso di un'immagine che sintetizza il senso di crepuscolo e di fine di un'età mitica: 
[...] haciendo tiempo para llamar el sueño, deambuló por las calles desiertas y la playa. Arriba el cielo se mantenía despejado ahora con todas sus estrellas. A sus pies, la marea iba borrando los nombres de todos los niños, escritos uno tras otro en la arena, que como cada año rubricaban con sus trazos inciertos la despedida, el adiós al último día del verano (Fernández Santos 1983: 190).

Chi ha meglio sondato la profondità del debito dell'autore spagnolo verso Pavese è, senza dubbio, il critico José Carlos Mainer (Mainer 1994: 48-52): le caratteristiche che siamo andati enucleando dal racconto (che, va detto, sporge sul futuro dell'opera dell'autore, sugli ambienti borghesi, e sulle tematiche che verranno affrontate nel romanzo Laberintos, più di quanto non facciano le narrazioni precedenti, maggiormente prossime ai romanzi Los bravos e En la Hoguera) le troviamo segnalate nelle pagine dedicate alla raccolta di Fernández Santos:

[...] hay un montón de cosas que remiten los cuentos de Cabeza rapada a la modalidad que el italiano llamó, con frase feliz, immagine-racconto (imagen-relato): la sensibilidad para cargar un clima físico, la capacidad para cargar de sentido un diálogo banal, la potenciación del gesto humano, el endiablado arte del elipsis y del final abierto o interrumpido [...] (Mainer 1994: 126).

Di tale fenomenologia espressiva può costituire un probante campione il finale, nella rapidità di uno scambio di battute dall'apparente corsiva insignificanza, del racconto Fiestas:

Amaneciendo despertó. Los compañeros se habían ido poco a poco retirando. Ante él, una borrosa silueta cuya voz reconoció, dijo. Anda, levántate. ¿Qué pasa? Que vayas a acostarte. Las piernas le dolían, envaradas, entumecidas. Tienes que acostarte -repitió el padre- si no mañana no vas a tirar del cuerpo. Será hoy.Y los dos, serios, casi desconocidos de nuevo el uno para el otro, marcharon despacio, en la luz amarillenta de la mañana, rumbo a casa (Fernández Santos 1983: 16).

Nella sommessa e discreta gravità di poche righe viene sancito il passaggio all'età adulta di Antonio, il personaggio principale, protagonista, insieme ad alcuni compagni, di un furto di una caraffa di vino, seguito da una fuga per i campi, dal marcato simbolismo mitico-iniziatico, rafforzato dalle circostanza rituale di una festa per la fine del raccolto e dal bagno notturno, in un contesto naturale dalla fisionomia fortemente antropomorfica:

Antonio mira el río ante sí. El verdín de las aguas ondulaba en el fondo. Parecía las oscuras manos del agua. Y el agua reflejaba, al resplandor del fuego, las escuálidas siluetas de los muchachos en calzoncillos, saltando sobre el césped (Fernández Santos 1983: 14).

Nel ritmo avventuroso e nel tono di favolosa scoperta del mondo extraterritoriale a cui si accede attraverso un'infrazione, il racconto, al solito modo concentrato, molto scenico e dalla rigorosa scansione di una sintassi rastremata, riporta alla 
mente quello pavesiano de $\mathrm{Il}$ mare, ugualmente costruito su di una fuga portata a compimento da protagonisti adolescenti.

Il fatto è che le coincidenze tra i due autori non si limitano al trattamento formale della materia, ma, come si è andati accennando, sono della materia stessa, della predilezione tematica per le età liminari, e per la trasfigurante e dolorosa dolcezza dei loro rapporti umani (mi riferisco, in particolare, all'atteggiamento di ammirata competitività verso il cugino del protagonista di El primo Rafael, e alla relazione giovane-adulto di Hombres), nella cui configurazione i due autori sembrano leggere, metonimicamente, il senso di un destino: 1'aneddotica episodicità di piccoli fatti nasconde, spesso, i termini di una poderosa allegoria interiore, in cui si condensa tutta una visione della vita. Anche su questa falda tematica ha messo l'occhio Mainer il quale, concentrando la propria attenzione critica su Día de caza, ha messo in luce come i costituenti di questo racconto ricordino, in forma singolare, quelli del componimento inaugurale di Lavorare stanca, I mari del sud. Nella visione e delusione finale, nella riduzione dell' animale a qualcosa di «tanto pacifico» da far sorridere il bambino, nella degradazione del mito, è forse dato sentire una nota simile a quella che Pavese tocca nel racconto $L$ ' eremita, sfociante in una simile parabola di conoscenza e addomesticamento banalizzante del perturbante. Sull'importanza storica della silloge di Fernández Santos «que significó mucho en el afianzamiento de la estética-y la ética- del realismo objetivo», si è pronunciato ancora Mainer:

[...] su publicación en la Biblioteca Breve de Seix-Barral (aquella cubierta tan einaudiana .... ) sellaba un propósito y un programa: Barral y sus amigos habían publicado ya a Cesare Pavese, el nouveau roman, La hora del lector de Castellet [...] Este libro significaba una apertura al grupo realista de Madrid, fraguado en torno a la Revista Española, y la fecha lo lo haría hermano de prensas e intenciones del mejor texto neorrealista del grupo barcelonés: Las afueras de Luis Goytisolo, ganador del primer premio Biblioteca Breve. Algo une a todos: la estética de lo implícito, la intensidad con que la mirada debe reemplazar a la descripción intencionada [...] Se piensa automáticamente en Cesare Pavese (Mainer 1994: 212).

parole che, in sintesi, descrivono l'atmosfera intellettuale del tempo, un clima culturale, caratterizzato dall'egemonia di un'estetica onnipervasiva, dominante, che raccoglie e accomuna i due centri culturali piu importanti del paese, Barcellona e Madrid, nella "compattezza" di una generazione. Se evidenti fili rossi legano gli autori spagnoli che siamo andati trattando, formatisi negli anni cinquanta, e stretti da legami amicali, cenacoli letterari, fitti scambi di lettura e, spesso, unità di propositi estetici ed eredità culturali, più dispersa, occasionale e frantumata fatta di avventure individuali, di iniziative intellettuali autonome, pare essere la ricezione creativa-critica di Pavese a partire, soprattutto dalla seconda metà degli anni sessanta.

Altri erano i venti culturali che tiravano, le estetiche oggettive e realistiche vennero soppiantate da quelle che insistevano sull'autonomia del fatto letterario, sulla sua specificità: metaromanzo e metapoesia, sperimentalismo e ludismo linguistico divennero concetti cardine, parole d'ordine di una Spagna che veniva rapidamente recuperando il gap che la divideva dagli altri paesi europei. Pavese perderà il protagonismo di cui aveva goduto, adombrato e dal massiccio afflusso dei latinoameri- 
cani (Cortázar e Borges, in primis) e dalla ripresa di altri europei (Joyce e Beckett), in una panorama in cui sulla scena letteraria si accavalleranno e incrocieranno tendenze diverse, in una caotica mescolanza e generale equivalenza, il nome di Pavese emergerà solo in bocca di chi, come Luis Mateo-Díez, scommette su di un realismo metaforico capace di parlare della condizione dell'uomo contemporaneo rappresentando, paradossalmente, un piccolo mondo periferico, affondando nel particolare geografico di una provincia per disegnare il diagramma dell'universale umano.

\section{Bibliografía}

BRAVO, María Elena (1985): Faulkner en España. Barcelona, Península.

BASILE, Bruno (1985): La finestra socchiusa. Bologna, Patron.

CORRALES EgEA, José (1971): La novela española actual. Madrid, Cuadernos para el diálogo.

CASTELLET, José María (1957): La hora del lector. Barcelona, Seix Barral.

FERNÁNDEZ FERNÁNDEZ, Luis Miguel (1992): El neorrealismo en la narración española de los años cincuenta. Santiago de Compostela, Universidade de Santiago de Compostela.

FERNÁNDEZ SANTOS, Jesús (1983): Cabeza rapada. Madrid, Planeta.

GIMFERRER, Pere (1978): Introducción a Obras Completas de Juan Goytisolo. Madrid, Aguilar, pp. 9-64.

GoYTisolo, Juan (1959): Problemas de la novela. Barcelona, Seix Barral.

GoYTisolo, Juan (1981): Obras completas. Madrid, Aguilar.

GoYTISOLO, Juan (1992): En los reinos de Taifa. Barcelona, Seix Barral.

GoYTISOLO, Luis (1996): Las afueras. Madrid, Espasa Calpe.

GoYTisolo, Luis (1961): Las mismas palabras. Barcelona, Seix Barral.

JORDAN, Barry (1990): Writing and politics in Franco's Spain. New York, Routledge.

LÁZARO, Jesús (1982): Juan Goytisolo. Madrid, Ministerio de Cultura.

MAINER, José Carlos (1994): De posguerra. Barcelona, Crítica.

SANZ VILlanUeVA, Santos (1980): Historia de la novela social española. Alhambra, Editorilla.

SOBEJANO, Gonzalo (1970): Novela española de nuestro tiempo (en busca del pueblo perdido). Madrid, Editorial Prensa Española. 